\title{
Adaptive Blind Source Separation and Equalization for Multiple-Input/Multiple-Output Systems
}

\author{
Ye (Geoffrey) Li, Senior Member, IEEE, and K. J. Ray Liu, Senior Member, IEEE
}

\begin{abstract}
In this paper, we investigate adaptive blind source separation and equalization for multiple-input/multiple-output (MIMO) systems. We first analyze the convergence of the constant modulus algorithm (CMA) used in MIMO systems (MIMOCMA). Our analysis reveals that the MIMO-CMA equalizer is able to recover one of the input signals, remove the intersymbol interference (ISI), and suppress the other input signals. Furthermore, for the MIMO finite impulse response (FIR) systems satisfying certain conditions, the MIMO-CMA FIR equalizers are able to perfectly recover one of the system inputs regardless of the initial settings. We then propose a novel algorithm for blind source separation and equalization for MIMO systems. Our theoretical analysis proves that the new blind algorithm is able to recover all system inputs simultaneously regardless of the initial settings. Finally, computer simulation examples are presented to confirm our analysis and illustrate the effectiveness of blind source separation and equalization for MIMO systems.
\end{abstract}

Index Terms - Blind equalization, convergence, multiple-input/ multiple-output system, source separation.

\section{INTRODUCTION}

I $\mathrm{N}$ many systems, the received or observed signals are superpositions of several linearly distorted signals from different sources. The systems in these scenarios can be modeled as multiple-input multiple-output (MIMO) systems. Examples of MIMO systems include spatial division multiple access (SDMA) in wireless communications, speech processing, seismic exploration, and some of biological systems. One of the most crucial problems for MIMO systems is not only to separate these signals, but also to compensate for the system distortion. In this paper, we will investigate blind source separation and equalization for MIMO systems, which is especially useful when the parameters of MIMO systems are not available.

For single-input/single-output (SISO) systems, many blind identification algorithms [5], [22], [33] and equalization algorithms [3], [8], [25], [26], [29], [30], [39] have been proposed by exploiting statistics of the system output. The Godard algorithm (GA) [8], also known as the constant modulus algorithm (CMA) [29], [30], is perhaps the best known and the simplest among various adaptive blind algorithms. It has been

Manuscript received March 7, 1997; revised June 1, 1998. This work was supported in part by the NSF under Grants MIP9309506 and MIP9457397. The material in this paper was presented in part at the ICC'96, Dallas, TX, June 1996.

Y. (G.) Li is with AT\&T Labs-Research, Red Bank, NJ 07701-7033 USA (e-mail: liye@ research.att.com).

K. J. R. Liu is with the Electrical Engineering Department, University of Maryland, College Park, MD 20742 USA (e-mail: kjrliu@ src.umd.edu).

Publisher Item Identifier S 0018-9448(98)06893-X shown [6], [26] that, for double infinite-length equalizers, the CMA will always converge to a global minimum regardless of initial settings. The local convergence properties of the CMA, when implemented with finite impulse response (FIR) equalizers, have been observed and analyzed in the literature, such as [4], [13], [28] and the references therein.

Single-input/multiple-output (SIMO) systems can be viewed as fractionally sampled communication systems or antenna arrays receiving multiple distorted versions of the same input signal. The fractionally spaced equalizer [7], [36] was originally proposed to suppress the timing sensitivity of communication systems. The convergence performance of the fractionally spaced decision-feedback equalizer has been investigated in [19]. If the parameters of SIMO systems are unknown, then the Godard algorithm, or the CMA, can also be used for adaptive blind system equalization. The convergence of the fractionally spaced CMA (FS-CMA) adaptive blind equalizer has been studied in [11] and [14]. Recently, the fractionally spaced CMA adaptive blind equalizer under symbol timing offsets has also been considered in [34].

The optimum MIMO equalization is studied in [24] and [42] when the parameters of MIMO systems are known. When the parameters of MIMO systems are not available, however, blind techniques have to be used to identify MIMO systems explicitly or implicitly in order to separate sources and equalize system distortion. Blind parameter estimation based on second-order statistics has been investigated in [1], [2], [15], [17], [18], and [27] for FIR systems and in [9] and [27] for ARMA systems. Blind parameter estimation can also exploit higher order statistics [40], [41], [43], or the finite-alphabet property of system inputs [32], [38], [37].

The CMA blind equalizer can also be used in MIMO systems. The capture properties of the CMA algorithm for MIMO systems with constant modulus input signals have been investigated in [16], [18], [21], [30], [31], and [35]. In [16] and [18] we also proposed a novel algorithm for simultaneous blind source separation and equalization. Recently, a CMA-based algorithm has been presented in [23], however, its convergence properties are not clear.

In this paper, we will introduce our research results on adaptive blind source separation and equalization for MIMO systems, part of which has already been presented [16], [18]. We first discuss the capture ability of the CMA blind equalizer for MIMO systems. Our analysis demonstrates that the MIMO-CMA blind equalizers are able to recover one of the input signals, while suppressing the other signals. Furthermore, under certain condition, the MIMO-CMA FIR 


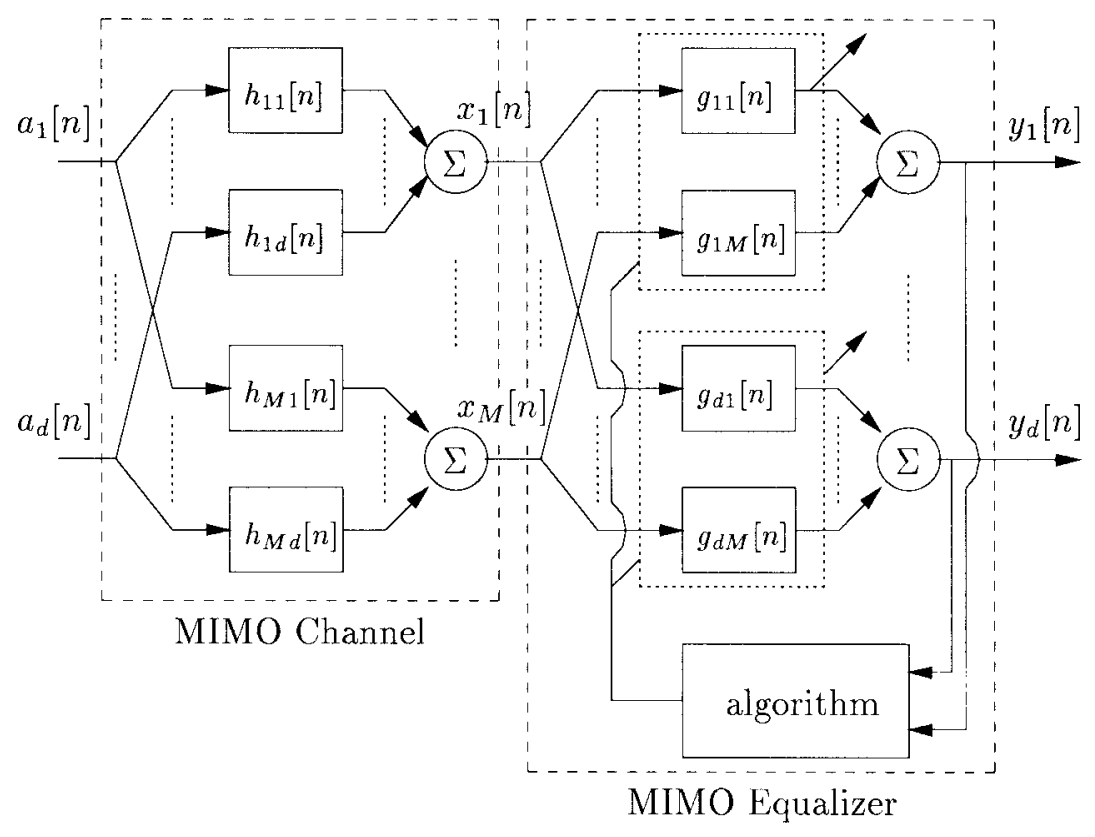

Fig. 1. Multiple-input and multiple-output system and equalizer.

equalizers, regardless of initial settings, can perfectly recover one of the input signals. Our analysis has extended the results in [21], [30], and [31]. Then, we develop a novel algorithm for adaptive blind equalization of MIMO systems. Theoretical analysis and computer simulation demonstrate that the new algorithm is able to recover all the input signals simultaneously while at the same time, removing ISI.

The remaining part of this paper is organized as follows. In Section II, we formulate the problem of blind source separation and equalization for MIMO systems and introduce a necessary and sufficient condition for an MIMO system to have a bounded-input and bounded-output (BIBO) stable equalizer that can achieve distortionless reception. Then in Section III, we analyze the convergence of the MIMO-CMA blind equalizer, and reveal many good convergence properties of the MIMO-CMA equalizers. Next, in Section IV, we develop a novel blind equalization algorithm to recover all input signals simultaneously, and then, we prove the global convergence of the new algorithm in Section V. Finally, we present computer simulations to confirm our analysis and illustrate the performance of the new algorithm in Section VI.

\section{Blind Adaptive Source Separation AND EQUALIZATION FOR MIMO SYSTEMS}

An MIMO linear system is shown in Fig. 1, where the additive noise has been ignored. The $d$ complex sequences $a_{1}[n], \cdots, a_{d}[n]$ are sent through different linear systems with impulse responses $h_{i j}[n]$ for $i=1, \cdots, M$ and $j=1, \cdots, d$ $(d \leq M)$. The system input sequences here are assumed to satisfy

$$
E\left\{a_{i}[n]\right\}=E\left\{a_{i}^{2}[n]\right\}=0
$$

and

$$
2 m_{2}^{2}-m_{4}>0
$$

where

$$
E\left\{\left|a_{i}[n]\right|^{2}\right\} \triangleq m_{2} \quad E\left\{\left|a_{i}[n]\right|^{4}\right\} \triangleq m_{4} .
$$

Equation (2) requires that the distributions of the system input sequences have small tails, which is true for subGaussian random variables [3] and almost all signals in digital communication systems. If we define the system output vector $\boldsymbol{x}[n]$, the system impulse response matrix $H[n]$, and the system input vector $a[n]$ as

$$
\boldsymbol{x}[n] \triangleq\left(\begin{array}{c}
x_{1}[n] \\
\vdots \\
x_{M}[n]
\end{array}\right) \quad H[n] \triangleq\left(\begin{array}{ccc}
h_{11}[n] & \cdots & h_{1 d}[n] \\
\vdots & \vdots & \vdots \\
h_{M 1}[n] & \cdots & h_{M d}[n]
\end{array}\right)
$$

and

$$
\boldsymbol{a}[n] \triangleq\left(\begin{array}{c}
a_{1}[n] \\
\vdots \\
a_{d}[n]
\end{array}\right)
$$

respectively, then, similar to SISO systems, the input-output relation for MIMO systems can be expressed as

$$
\boldsymbol{x}[n]=H[n] * \boldsymbol{a}[n]
$$

where $*$ denotes the convolution of the matrix (or vector) sequences. For general matrix sequences $\left(a_{i j}[n]\right)$ and $\left(b_{i j}[n]\right)$, their convolution is defined as

$$
\left(a_{i j}[n]\right) *\left(b_{i j}[n]\right) \triangleq\left(\sum_{k} a_{i k}[n] * b_{k j}[n]\right) .
$$

Equation (5) can also be written in $Z$-domain as

$$
\boldsymbol{x}(z)=H(z) \boldsymbol{a}(z)
$$

where $\boldsymbol{x}(z), \boldsymbol{a}(z)$, and $H(z)$ are the $Z$-transform of $\boldsymbol{x}[n], \boldsymbol{a}[n]$, and $H[n]$, respectively. For MIMO FIR systems, $H(z)$ is a polynomial matrix. 
As in Fig. 1, to recover the system input $\boldsymbol{a}[n]$, a linear equalizer is applied to the system output $\boldsymbol{x}[n]$ to achieve distortionless reception. That is, the impulse response matrix sequence of the linear equalizer, $G[n]$, satisfies

$$
G[n] * H[n]=\delta[n] I_{d}
$$

or, in the $Z$-domain,

$$
G(z) H(z)=I_{d}
$$

where $I_{d}$ is a $d \times d$ identity matrix. $G[n]$ is defined as

$$
G[n] \triangleq\left(\begin{array}{ccc}
g_{11}[n] & \cdots & g_{1 M}[n] \\
\vdots & \cdots & \vdots \\
g_{d 1}[n] & \cdots & g_{d M}[n]
\end{array}\right)
$$

and $G(z)$ is the $Z$-transform of $G[n]$. Initially, we may take the filters for equalization in Fig. 1 as being bounded-input and bounded-output (BIBO) stable, however, potentially noncausal (double infinite length) so as to deal with the MIMO systems with noncausal inverses.

In blind source separation and equalization, the original sequences $a_{i}[n]$ 's for $i=1, \cdots, d$ are unknown to the receivers except for their statistical properties. Usually, the statistics of the systems are the same. Thus the recovered signals from blind equalization will be subject to phase and permutation ambiguity. Therefore, a desirable property of MIMO blind equalizers should be

$$
G(z) H(z)=P D(z)
$$

where $P$ is a $d \times d$ permutation matrix and $D(z)$ is a diagonal matrix defined as

$$
D(z)=\operatorname{diag}\left\{e^{\jmath \theta_{1}} z^{-n_{1}}, \cdots, e^{\jmath \theta_{d}} z^{-n_{d}}\right\}
$$

where $\theta_{i} \in[-\pi, \pi]$ and $n_{i}$ is an integer for $i=1, \cdots, d$. The equalizers with $G(z)$ satisfying (11) are called the distortionless reception equalizer for system $H(z)$. It is obvious that the distortionless reception equalizer for a given MIMO system is not necessarily unique because of the multiple choices of $P$, $\theta_{i}$, and $n_{i}$ in (11) and (12).

An MIMO system is said to satisfy the distortionless reception condition if there exists a $\mathrm{BIBO}$ stable distortionless reception equalizer for this system. Not all systems have a BIBO stable distortionless reception equalizer. It is well known that an SISO system satisfies the distortionless reception condition if and only if the $Z$-transform of the system impulse response has no zero on the unit circle. For MIMO systems, the following theorem gives a necessary and sufficient condition.

Theorem 1: There exists a BIBO stable, linear, and distortionless reception equalizer for an MIMO system if and only if $H^{\dagger}\left(e^{\jmath \omega}\right) H\left(e^{\jmath \omega}\right)$ is nonsingular for all $\omega \in[-\pi, \pi]$.

Proof: If $H^{\dagger}\left(e^{\gamma \omega}\right) H\left(e^{\jmath \omega}\right)$ is nonsingular for all $\omega \in$ $[-\pi, \pi]$, then it is invertible and

$$
G\left(e^{\jmath \omega}\right)=\left(H^{\dagger}\left(e^{\jmath \omega}\right) H\left(e^{\jmath \omega}\right)\right)^{-1} H^{\dagger}\left(e^{\jmath \omega}\right)
$$

is the Fourier transform of a BIBO stable equalizer satisfying (11).

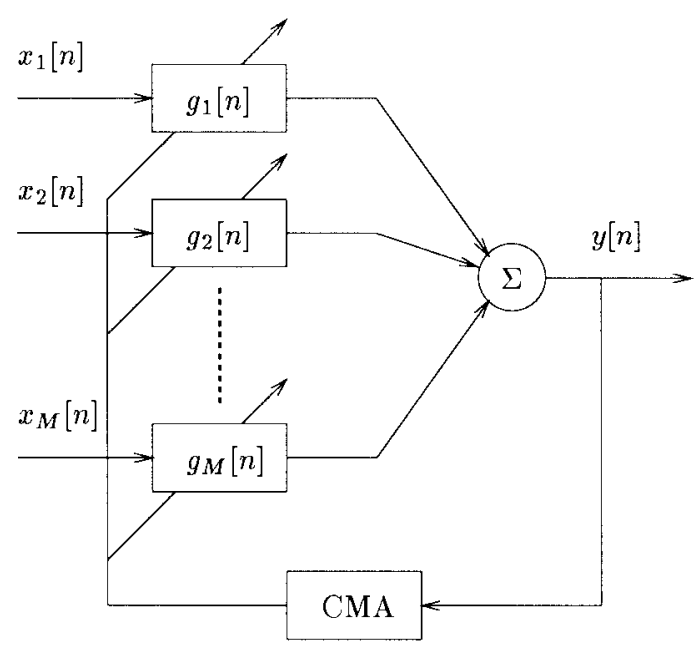

Fig. 2. The MIMO-CMA blind equalizer.

Conversely, if $H^{\dagger}\left(e^{j \omega_{0}}\right) H\left(e^{j \omega_{0}}\right)$ is singular for some $\omega_{0} \in$ $[-\pi, \pi]$, then $H\left(e^{\pi_{0}}\right)$ will not be of full-rank. If there is a $G\left(e^{\jmath \omega}\right)$ satisfying (11), then

$$
P D\left(e^{\jmath \omega_{0}}\right)=G\left(e^{\jmath \omega_{0}}\right) H\left(e^{x \omega_{0}}\right)
$$

would be singular. This is a contradiction since $P D\left(e^{\varkappa_{0}}\right)$ is nonsingular from its definition. Therefore, there is no BIBO stable, linear, and distortionless equalizer in this case.

From Theorem 1, a necessary and sufficient condition for an MIMO system to have a distortionless reception equalizer is that $H\left(e^{x \omega}\right)$ is of full rank for all $\omega \in[-\pi, \pi]$, and $M \geq d$, that is, the number of system outputs is no less than the number of system inputs. In what follows, we will always assume that the discussed MIMO systems satisfy this condition.

\section{Capture Properties of the MIMO-CMA Equalizer}

In this section, we will investigate convergence of the CMA equalizer used in MIMO systems. The MIMO-CMA blind equalizer discussed in this section is shown in Fig. 2. After each system output, a linear BIBO stable filter is used. The filter coefficients $g_{m}[n]$ are adjusted to minimize the Godard cost function [8], [29], [30]

$$
C(y[n])=\frac{1}{4} E\left\{\left(|y[n]|^{2}-r\right)^{2}\right\}
$$

where $r$ is the dispersion constant of the MIMO system inputs, defined as

$$
r=\frac{m_{4}}{m_{2}} .
$$

\section{A. General Convergence of MIMO-CMA Blind Equalizer}

From Fig. 2, the equalizer output can be expressed as

$$
y[n]=\sum_{i=1}^{d} \sum_{k=-\infty}^{\infty} a_{i}[n-k] s_{i}[k]
$$

where $s_{i}[n]$ is the impulse response of the equalized system corresponding to the $i$ th input signal that is related to $h_{m i}[n]$ 
and $g_{m}[n]$ by

$$
s_{i}[n]=\sum_{m=1}^{M} \sum_{k=-\infty}^{\infty} h_{m i}[k] g_{m}[n-k] .
$$

With (17), the Godard cost function defined in (15) can be expressed, in terms of $s_{i}[n]$ 's, as

$$
\begin{aligned}
& C(y[n]) \\
& =\frac{1}{4}\left[-\left(2 m_{2}^{2}-m_{4}\right) \sum_{i, n}\left|s_{i}[n]\right|^{4}+2 m_{2}^{2}\left(\sum_{i, n}\left|s_{i}[n]\right|^{2}\right)^{2}\right. \\
& \left.\quad-2 m_{4} \sum_{i, n}\left|s_{i}[n]\right|^{2}+m_{4}^{2} / m_{2}^{2}\right]
\end{aligned}
$$

If we denote

$$
\boldsymbol{s} \triangleq\left(\cdots, s_{d}[-1], s_{1}[0], \cdots, s_{d}[0], s_{1}[1], \cdots\right)
$$

then the Godard cost function (19) is a functional of $\boldsymbol{s}$, which has a similar form to that of the CMA equalizer for SISO systems [6], [13]. Hence, if the MIMO system satisfies the distortionless reception condition, and the length of the equalizer in Fig. 2 is double-infinite, following Foschini's arguments [6], it can be easily shown that the only minimum points of the MIMO-CMA equalizer in Fig. 2 are

$$
\left|s_{i}[n]\right|^{2}=\delta\left[n-n_{d}, i-i_{0}\right] \text {, for some integers } n_{d} \text { and } i_{0}
$$

where $\delta[n, i]$ is defined as

$$
\delta[n, i] \triangleq \begin{cases}1, & \text { if } n=0 \text { and } i=0 \\ 0, & \text { otherwise. }\end{cases}
$$

This implies that the MIMO-CMA equalizer is able to recover one of the system inputs with a time delay and phase ambiguity and suppress the other input signals.

For the MIMO systems with constant modulus input signals, the above capture property has been proved in [21], [30], and [31]. Our discussion here indicates that this capture property is preserved for the MIMO systems with any inputs satisfying the assumed condition in Section II. Furthermore, the other convergence properties are also preserved. Before stating these properties, we first give some relevant definitions.

The attainable set $S_{a}$ for a given (finite or infinite) equalizer is defined as

$$
\begin{aligned}
S_{a} \triangleq & \left\{s: s_{i}[n]=\sum_{m=1}^{M} \sum_{k} h_{m i}[n-k] g_{m}[k],\right. \\
& \left.\sum_{k}\left|g_{m}[k]\right|<\infty\right\}
\end{aligned}
$$

In the above definition, the range of $k$ relies on the length of the equalizer. It is obvious that $S_{a}$ depends on the parameters of the MIMO systems.
The unique global minimum set cone $S_{i, n}$ is defined as

$$
\begin{gathered}
S_{i, n} \triangleq\left\{s:\left|s_{i}[n]\right|>\left|s_{j}[k]\right| \text { for all } i \neq j \text { or } n \neq k\right. \\
\text { and } \left.\sum_{j, k}\left|s_{j}[k]\right|<\infty\right\},
\end{gathered}
$$

and the boundary of $S_{i, n}, B_{i, n}$, is defined as

$$
\begin{gathered}
B_{i, n} \triangleq\left\{s:\left|s_{i}[n]\right|=\left|s_{j}[k]\right| \text { for some } i \neq j \text { or } n \neq k\right. \\
\text { and } \left.\sum_{j, k}\left|s_{j}[k]\right|<\infty\right\} .
\end{gathered}
$$

With the above definitions, we can state the general convergence properties of the finite-length MIMO-CMA equalizers as follows.

Theorem 2: Let $S_{a}$ be the attainable set of a given finitelength MIMO-CMA equalizer.

i) If the initial equalizer parameters settings are such that the initial equalized system impulse response vector $\boldsymbol{s}^{\text {in }} \in S_{a} \cap S_{i, n}$ and its output satisfies the kurtosis condition

$$
\frac{\operatorname{kurt}(y[n])}{\operatorname{kurt}(a[n])}>0.5
$$

then for a sufficiently small step size, the equalizer will cause $\boldsymbol{s}$ to converge to a minimum point inside $S_{a} \cap S_{i, n}$. In the above expression

$$
\operatorname{kurt}(x) \triangleq \frac{K(x)}{\sigma_{x}^{4}}
$$

where $\sigma_{x}^{2}$ is the variance of $x$ and $K(x)=E\left\{|x|^{4}\right\}-2 \sigma_{x}^{4}$ is the kurtosis of complex random variable $x$ satisfying $E\left\{x^{2}\right\}=0$.

ii) Denoting

$$
\begin{gathered}
E_{i, n}=\left\{\boldsymbol{s}: s_{i}[n]=e^{\jmath \phi}, \phi \in[-\pi, \pi]\right. \text { and } \\
\left.s_{j}[k]=0 \text { if } j \neq i \text { or } k \neq n\right\}
\end{gathered}
$$

a) if $E_{i, n} \subset S_{a} \cap S_{i, n}$, then there is only one minimum set $E_{i, n} \subset S_{a} \cap S_{i, n}$ while there is no minimum point on the boundary of $S_{i, n}$;

b) if $E_{i, n}$ is near $S_{a} \cap S_{i, n}$, then there must exist only one minimum set in $S_{a} \cap S_{i, n}$ near $E_{i, n}$ while all other possible minima are near the boundary of $S_{i, n}$.

Theorem 2 is basically the generalization of [13, Theorems 6.2 and 6.3] for SISO systems, or [14, Theorem 3.2] for SIMO systems. Its proof is similar to that of [13, Theorems 6.2 and 6.3], therefore, it is omitted here.

According to Theorem 2 i), if we want to use the MIMOCMA equalizer to capture the $i$ th input signal, compensate for the system distortion, and suppress other sources, it is sufficient to select the initial setting of the equalizer such that $\boldsymbol{s}^{\text {in }} \in S_{i, n}$ for some $n$ and the initial system output satisfies the 
kurtosis condition, which is especially useful when we know a rough estimation of the system parameters.

Theorem 2 ii) indicates the locations of the minima of the MIMO-CMA equalizers. Based on this part of the theorem, the initialization strategy discussed in [6] and [13] can also be used for the MIMO-CMA blind equalizers.

\section{B. Global Convergence of MIMO-CMA FIR Equalizer}

In practice, most of the MIMO systems can be approximated as FIR systems. Without loss of generality, we can assume that the FIR system impulse responses satisfy

$$
h_{m i}[n]=0, \quad \text { for } n<0 \text { or } n>L
$$

for $i=1, \cdots, d$ and $m=1, \cdots, M$, where $L$ is the length of system impulse responses. The length of the impulse responses, $s_{i}[n]$ 's, of the equalized system is $L+K-1$ if an MIMO-CMA FIR equalizer with length $K$ is used for an MIMO FIR system with length $L$. Let the parameters of the FIR equalizer be

$$
g_{m}[n]=0, \quad \text { for } n<0 \text { or } n>K
$$

for $m=1, \cdots, M$. The relationship between the equalizer parameters $g_{m}[n]$ and the impulse response of the equalized system $s_{i}[n]$ can be expressed as

$$
\boldsymbol{s}_{L+K-1}=\boldsymbol{g}_{K} \mathcal{H}_{K}
$$

where

$$
\begin{array}{r}
\boldsymbol{s}_{L+K-1} \triangleq\left(s_{1}[0], \cdots, s_{d}[0], \cdots, s_{1}[L+K-2], \cdots\right. \\
\left.s_{d}[L+K-2]\right) \\
\boldsymbol{g}_{K} \triangleq\left(g_{1}[0], \cdots, g_{M}[0], \cdots, g_{1}[K-1], \cdots, g_{M}[K-1]\right)
\end{array}
$$

and (33) at the bottom of this page. The singularity of the generalized Sylvester matrix $\mathcal{H}_{K}$ plays a crucial role in the convergence of the MIMO-CMA FIR equalizer. The relationship between the rank of $\mathcal{H}_{K}$ and the reducibility of $H(z)$ has been studied in the multivariable control literature, such as in [10]. Before introducing the relationship, we first give the definition of the irreducibility of a matrix polynomial.

Definition: An $M \times d(M>d)$ polynomial matrix $H(z)$ is said to be irreducible [10] if there is no $d \times d$ polynomial matrix $R(z)$ with nonconstant $\operatorname{det}(R(z))$, such that $H(z)=$ $\tilde{H}(z) R(z)$, where $\tilde{H}(z)$ is an $M \times d$ polynomial matrix.

Using the results in [10], the following lemma can be proved [1], [2], [35].
Lemma 1: Let $H[L-1]$ be of full column-rank, then $\mathcal{H}_{K}$ is of full column-rank for all $K \geq d L-1$, if and only if $H(z)$ is irreducible.

With the above lemma, we are able to prove the following global convergence theorem for the CMA FIR equalizers used in MIMO FIR systems.

Theorem 3: For an MIMO FIR system of length $L$, if $H(z)$ is irreducible with $H[L-1]$ being of full rank, then any MIMO-CMA FIR blind equalizer with length $K \geq d L-1$ can achieve global convergence regardless of its initial setting.

Proof: Since $\mathcal{H}_{K}$ is of full rank for all $K \geq d L-1$ from Lemma 1, therefore, $\mathcal{H}_{K}^{\dagger} \mathcal{H}_{K}$ is invertible, and for any $\boldsymbol{s}_{L+K-1} \in \mathcal{C}^{d(L+K-1)}$, there exists

$$
\boldsymbol{g}_{K}=\boldsymbol{s}_{L+K-1}\left(\mathcal{H}_{K}^{\dagger} \mathcal{H}_{K}\right)^{-1} \mathcal{H}_{K}^{\dagger}
$$

such that $\boldsymbol{s}_{L+K-1}=\boldsymbol{g}_{K} \mathcal{H}_{K}$. Hence, $E_{i, n}$ 's for $i=1, \cdots, d$ and $n=0, \cdots, L+K-2$ are in attainable set $S_{a}$. From Theorem 2 ii) a), the only minimum set of the MIMO-CMA FIR equalizer in $S_{i, n}$ is $E_{i, n}$, and there is no minimum on the boundary of $S_{i, n}$. Since $S_{a} \cap S_{i, n}$ is empty for $i=1, \cdots, d$ and $n<0$ or $n>L+K-2$, the MIMO-CMA FIR equalizer has no other (local) minimum. Hence, all minima of the MIMO-CMA FIR equalizer in this case are global ones. Therefore, regardless of the initial setting, the equalizer will converge to one of its global minima.

The above theorem illustrates a very nice convergence property of the MIMO-CMA FIR equalizer. It indicates that the MIMO-CMA FIR equalizer can recover one of the system inputs, compensate for system distortion, and suppress the signals from the other sources if the MIMO-CMA FIR equalizer is long enough and the system satisfies the condition in Lemma 1.

\section{A New BLIND AlgorithM}

We have revealed the capture properties of the MIMO-CMA blind equalizer in the previous section. In this section, we will propose a new blind algorithm for MIMO systems, based on the CMA, to recover all system inputs and compensate for the system distortion. Without lost of generality, we will assume $d=2$ in this section. The algorithm developed in this section can be easily extended to $d>2$ systems.

\section{A. New Cost Function}

Consider the blind equalizer shown in Fig. 1. If we adjust the equalizer parameters for each individual system to minimize the Godard cost function in (15), then according to the analysis in Section III, the equalizer outputs $y_{1}[n]$ and $y_{2}[n]$ will

$$
\mathcal{H}_{K} \triangleq\left(\begin{array}{ccccccc}
H[L-1] & H[L-2] & \cdots & H[0] & & & \mathbf{0} \\
& H[L-1] & \ddots & \ddots & H[0] & & \\
& & \ddots & \ddots & \ddots & \ddots & \\
\mathbf{0} & & & H[L-1] & \ddots & \ddots & H[0]
\end{array}\right)
$$


TABLE I

An Adaptive Blind Algorithm for MIMO Systems

$<\left|y_{2}[n-l]\right|^{2}>_{n}=(1-\epsilon)<\left|y_{2}[n-1-l]\right|^{2}>_{n-1}+\epsilon\left|y_{2}[n-l]\right|^{2}$
$<\left|y_{1}[n-l]\right|^{2}>_{n}=(1-\epsilon)<\left|y_{1}[n-1-l]\right|^{2}>_{n-1}+\epsilon\left|y_{1}[n-l]\right|^{2}$
$<y_{1}[n] y_{2}^{*}[n-l]>_{n}=(1-\epsilon)<y_{1}[n-1] y_{2}^{*}[n-1-l]>_{n-1}+\epsilon y_{1}[n] y_{2}^{*}[n-l]$
$<y_{2}[n] y_{1}^{*}[n-l]>_{n}=(1-\epsilon)<y_{2}[n-1] y_{1}^{*}[n-1-l]>_{n-1}+\epsilon y_{2}[n] y_{1}^{*}[n-l]$
$z_{1}[n]=\sum_{l=0}^{\infty}\left(\left|y_{2}[n-l]\right|^{2} y_{1}[n]-<\left|y_{2}[n-l]\right|^{2}>_{n} y_{1}[n]-<y_{1}[n] y_{2}^{*}[n-l]>_{n} y_{2}[n-l]\right)$
$z_{2}[n]=\sum_{l=0}^{\infty}\left(\left|y_{1}[n-l]\right|^{2} y_{2}[n]-<\left|y_{1}[n-l]\right|^{2}>_{n} y_{2}[n]-<y_{2}[n] y_{1}^{*}[n-l]>_{n} y_{1}[n-l]\right)$
$g_{j m}^{(n)}[k]=g_{j m}^{(n-1)}[k]-\mu\left(\left(\left|y_{j}[n]\right|^{2}-r\right) y_{j}[n]-c_{o} z_{j}[n]\right) x_{m}^{*}[n-k]$

be from one of the two sources. Note that $y_{1}[n]$ and $y_{2}[n]$ may be from the same or different sources, depending on the equalizer's initial settings. Hence, to develop a blind algorithm that can recover all systems inputs, we only need to modify the Godard cost function, such that the outputs of the MIMO equalizer are from different sources.

The proposed cost function for adaptive blind equalization of MIMO systems is given by

$$
C_{\mathrm{MIMO}} \triangleq C\left(y_{1}[n]\right)+C\left(y_{2}[n]\right)-c_{O} K\left(y_{1}, y_{2}\right)
$$

where $c_{o} \geq m_{4} /\left(2 m_{2}^{2}-m_{4}\right)$, and $K\left(y_{1}, y_{2}\right)$ is a functional of $y_{1}(k)$ and $y_{2}(k)$ for all $k \leq n$ defined as

$$
\begin{aligned}
K\left(y_{1}, y_{2}\right) \triangleq & \frac{1}{2} \sum_{k=-\infty}^{-1} \operatorname{Cum}\left(y_{1}[n], y_{1}^{*}[n], y_{2}[n+k], y_{2}^{*}[n+k]\right) \\
& +\frac{1}{2} \sum_{k=0}^{\infty} \operatorname{Cum}\left(y_{1}[n-k], y_{1}^{*}[n-k], y_{2}[n], y_{2}^{*}[n]\right)
\end{aligned}
$$

with $\operatorname{Cum}\left(y_{1}, y_{1}^{*}, y_{2}, y_{2}^{*}\right)$ being the cumulant of the random complex variables $y_{1}, y_{1}^{*}, y_{2}$ and $y_{2}^{*}$, defined as

$$
\begin{aligned}
& \operatorname{Cum}\left(y_{1}, y_{1}^{*}, y_{2}, y_{2}^{*}\right) \\
& \triangleq E\left\{\left|y_{1}\right|^{2}\left|y_{2}\right|^{2}\right\}-E\left\{\left|y_{1}\right|^{2}\right\} E\left\{\left|y_{2}\right|^{2}\right\}-\left|E\left\{y_{1} y_{2}^{*}\right\}\right|^{2}
\end{aligned}
$$

for random variables $y_{i}$ satisfying

$$
E\left\{y_{i}\right\}=E\left\{y_{i}^{2}\right\}=0, \quad \text { for } i=1,2 .
$$

It can be seen from Lemma 2 of Section $\mathrm{V}$ that $K\left(y_{1}, y_{2}\right)=$ 0 if and only if the outputs of the equalizer are independent. Hence, $-c_{o} K\left(y_{1}, y_{2}\right)$ in (35) makes the blind algorithm converge to distinct sources. However, the estimation of $K\left(y_{1}, y_{2}\right)$ usually requires more data than that of $C\left(y_{i}[n]\right)$, therefore, the convergence speed of blind equalizer will be reduced.

In [23]

$$
\begin{aligned}
\tilde{K}\left(y_{1}, y_{2}\right)= & \sum_{k=0}^{\infty}\left|E\left\{y_{1}[n] y_{2}^{*}[n-k]\right\}\right|^{2} \\
& +\sum_{k=1}^{\infty}\left|E\left\{y_{1}[n-k] y_{2}^{*}[n]\right\}\right|^{2}
\end{aligned}
$$

has been proposed in the cost function of a blind algorithm. However, in that case, the convergence properties of the blind algorithm are unknown. The MIMO blind equalizer may have local minima besides the global ones.
Even though the cost function in (35) is valid only for $d=2$, for the $d>2$ case, it can be extended to

$$
C_{\mathrm{MIMO}}=\sum_{i=1}^{d} C\left(y_{i}[n]\right)-c_{o} \sum_{i, j=1}^{d} K\left(y_{i}, y_{j}\right)
$$

and the global convergence and local convergence properties discussed in the next section can be similarly generalized.

\section{B. Algorithm Development}

Using the stochastic gradient method to search for minimum points of the new cost function, we can implement the new algorithm as

$$
\begin{aligned}
g_{j m}^{(n)}[k]= & g_{j m}^{(n-1)}[k]-\mu\left(\left(\left|y_{j}[n]\right|^{2}-r\right) y_{j}[n]\right. \\
& \left.-c_{o} z_{j}[n]\right) x_{m}^{*}[n-k]
\end{aligned}
$$

for $j=1,2$ and $m=1, \cdots, M$, where $\mu$ is a small step size, $g_{j m}^{(n)}[k]$ is the $k$ th parameter of the $j m$ th filter after the $n$th iteration, and $z_{i}[n]$ 's are given by

$$
\begin{aligned}
z_{1}[n]= & \sum_{l=0}^{\infty}\left(\left|y_{2}[n-l]\right|^{2} y_{1}[n]-E\left\{\left|y_{2}[n-l]\right|^{2}\right\} y_{1}[n]\right. \\
& \left.-E\left\{y_{1}[n] y_{2}^{*}[n-l]\right\} y_{2}[n-l]\right) \\
z_{2}[n]= & \sum_{l=0}^{\infty}\left(\left|y_{1}[n-l]\right|^{2} y_{2}[n]-E\left\{\left|y_{1}[n-l]\right|^{2}\right\} y_{2}[n]\right. \\
& \left.-E\left\{y_{2}[n] y_{1}^{*}[n-l]\right\} y_{1}[n-l]\right) .
\end{aligned}
$$

If the ensemble average in the above expressions is substituted with the empirical average as in [26], the resulting algorithm can be expressed as in Table I.

The above developed algorithm is based on the stochastic gradient approach to search for the minima of $C_{\text {MIMO }}$. It is possible to develop an algorithm using Newton's algorithm [20] to find the minima of $C_{\mathrm{MIMO}}$, which will speed up the convergence, however, this will increase the computational complexity.

\section{Compound Blind Algorithm}

Based on the new blind algorithm, we can develop a compound algorithm to speed up the convergence. The compound algorithm contains the following three steps.

Step 1: Source Separation: Separating different sources using $K\left(y_{1}, y_{2}\right)$ defined in (46) as a cost function.

Step 2: Rough Equalization: Equalizing systems and separating sources using $C_{\text {MIMO }}$ defined in (35) as a cost function until the kurtosis condition (26) is satisfied for all equalizer outputs. 
Step 3: Fine Equalization: Adjusting the parameters of equalizer by means of the Godard cost function (15).

At the very beginning, the equalizer outputs contain signal components from different sources; hence, $K\left(y_{1}, y_{2}\right)$ in Step 1 is used for source separation. Once signal components from different sources are roughly separated, $C_{\mathrm{MIMO}}$ in Step 2 is used to further separate sources and at the same time remove intersymbol interference, such that all outputs of the blind equalizer satisfy the kurtosis condition. From Theorem 2 i), when all equalizer outputs satisfy the kurtosis condition, the blind equalizer using the Godard cost function will converge to desired parameter sets. Hence, only the Godard cost function is used in Step 3 to speed up the convergence.

\section{CONVERgence of the New Algorithm}

In the previous section, a new adaptive blind algorithm has been derived for source separation and equalization of MIMO systems. In this section, we first prove the global convergence of the new algorithm if the MIMO system and equalizer satisfy some mild conditions. And then, through an example, we demonstrate the ill-convergence of the new algorithm if certain conditions are not satisfied.

\section{A. Global Convergence}

Let the impulse response of the equalized system corresponding to the $i$ th source and the $j$ th output of the equalizer be

$$
s_{i j}[n] \triangleq \sum_{m=1}^{M} g_{j m}[n] * h_{m i}[n] .
$$

Then, the equalizer output can be written as

$$
y_{j}[n]=\sum_{i=1,2} \sum_{l=-\infty}^{\infty} a_{i}[l] s_{i j}[n-l]
$$

for $j=1,2$.

From the direct calculation in Appendix A, we have the following lemma.

Lemma 2: $K\left(y_{1}, y_{2}\right)$ defined in (36) can be expressed in terms of $s_{i j}[k]$ as

$$
K\left(y_{1}, y_{2}\right)=\frac{1}{2}\left(m_{4}-2 m_{2}^{2}\right) \sum_{i=1,2} \prod_{j=1,2}\left(\sum_{k}\left|s_{i j}[k]\right|^{2}\right) .
$$

Hence, $K\left(y_{1}, y_{2}\right) \leq 0$ if $2 m_{2}^{2}-m_{4}>0$.

From Lemma 2, $-K\left(y_{1}, y_{2}\right)$ attains its minimum, 0 , if and only if either

or

$$
s_{11}[n]=0 \quad \text { and } \quad s_{22}[n]=0 \quad \forall n
$$

$$
s_{12}[n]=0 \quad \text { and } \quad s_{21}[n]=0 \quad \forall n .
$$

In either case, the equalizer outputs $\left\{y_{1}[n]\right\}$ and $\left\{y_{2}[n]\right\}$ are independent. Hence, $K\left(y_{1}, y_{2}\right)$ in the cost function makes the new blind equalizer capable of separating different sources, as demonstrated by Theorem 4 .
However, $\tilde{K}\left(y_{1}, y_{2}\right)$ in (39) attains its minimum, 0, if and only if the system outputs $\left\{y_{1}[n]\right\}$ and $\left\{y_{2}[n]\right\}$ are uncorrelated, rather than independent. This is one of the reasons that we have chosen $K\left(y_{1}, y_{2}\right)$ in the cost function.

Using Lemma 2 and (19), the new cost function for the blind equalizer can be expressed in terms of $s_{i j}[n]$ as

$$
\begin{aligned}
C_{\mathrm{MIMO}}= & \frac{1}{4} \sum_{j=1,2}\left\{-\left(2 m_{2}^{2}-m_{4}\right) \sum_{i, n}\left|s_{i j}[n]\right|^{4}\right. \\
& \left.+2 m_{2}^{2}\left(\sum_{i, n}\left|s_{i j}[n]\right|^{2}\right)^{2}-2 m_{4} \sum_{i, n}\left|s_{i j}[n]\right|^{2}\right\} \\
& +\frac{1}{2} c_{o}\left(2 m_{2}^{2}-m_{4}\right) \sum_{i=1,2} \prod_{j=1,2}\left(\sum_{n}\left|s_{i j}[n]\right|^{2}\right) \\
& +\frac{1}{2} m_{4}^{2} / m_{2}^{2} .
\end{aligned}
$$

By means of the above expression, we are able to prove the global convergence of the new blind algorithm with the help of the following lemma that is proved in Appendix B.

Lemma 3: Let

$$
\begin{aligned}
f\left(t_{11},\right. & \left.t_{12}, t_{21}, t_{22}\right) \\
= & \frac{1}{4}\left\{-\left(2 m_{2}^{2}-m_{4}\right)\left(t_{11}^{2}+t_{12}^{2}+t_{21}^{2}+t_{22}^{2}\right)\right. \\
& +2 m_{2}^{2}\left[\left(t_{11}+t_{21}\right)^{2}+\left(t_{12}+t_{22}\right)^{2}\right] \\
& +2 c_{o}\left(2 m_{2}^{2}-m_{4}\right)\left(t_{11} t_{12}+t_{21} t_{22}\right) \\
& \left.-2 m_{4}\left(t_{11}+t_{12}+t_{21}+t_{22}\right)+2 m_{4} / m_{2}^{2}\right\} .
\end{aligned}
$$

For any

$$
c_{0} \geq m_{4} /\left(2 m_{2}^{2}-m_{4}\right)
$$

the only minima of $f\left(t_{11}, t_{12}, t_{21}, t_{22}\right)$ on $[0,+\infty)^{4}$ are

$\left(t_{11}, t_{12}, t_{21}, t_{22}\right)=(1,0,0,1)$ and

$$
\left(t_{11}, t_{12}, t_{21}, t_{22}\right)=(0,1,1,0) .
$$

The global convergence of the new blind equalization algorithm can be stated as follows.

Theorem 4: The MIMO blind equalizer using the cost function defined in (35) will converge to one of its global minimum points regardless of its initial setting, if the MIMO system and the equalizer satisfy one of the following two conditions.

i) the MIMO system satisfies the distortionless reception condition and an infinite-length MIMO filter is used as the equalizer; or

ii) $H(z)$, the $Z$-transform of the impulse response of the MIMO FIR system with length $L$, is an irreducible polynomial matrix with $H[L-1]$ being nonsingular, and the length of the MIMO equalizer $K \geq d L-1$.

Proof: i) Assume the MIMO system and equalizer satisfy condition i) in the theorem.

To find minimum of $C_{\mathrm{MIMO}}$, set

$$
\frac{\partial C_{\mathrm{MIMO}}}{\partial \bar{s}_{11}[n]}=0 \quad \forall n
$$



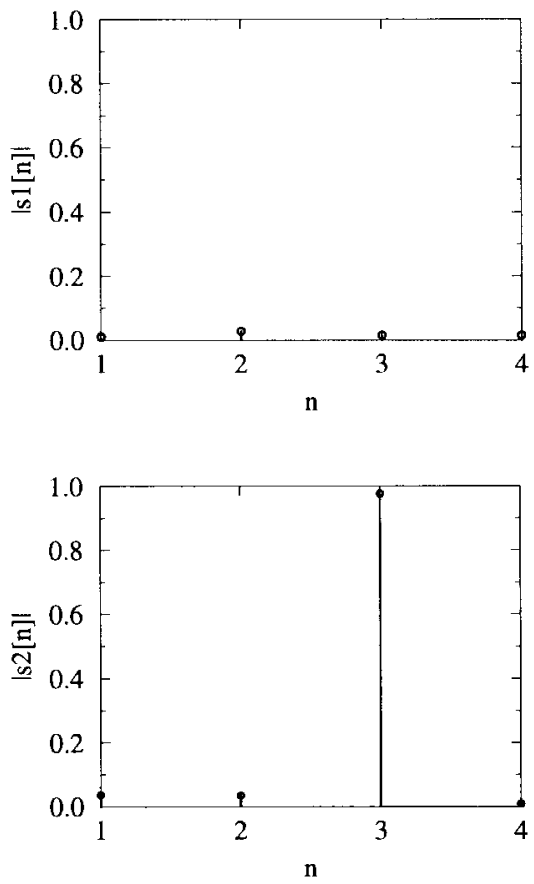

Fig. 3. The impulse responses of the equalized system after 10000 iterations.

where

$$
\frac{\partial}{\partial \bar{s}_{11}[n]} \triangleq \frac{1}{2} \frac{\partial}{\partial \operatorname{Re}\left\{s_{11}[n]\right\}}+\gamma \frac{1}{2} \frac{\partial}{\partial \operatorname{Im}\left\{s_{11}[n]\right\}} .
$$

Direct calculation yields

$$
\begin{aligned}
& -\left(2 m_{2}^{2}-m_{4}\right)\left|s_{11}[n]\right|^{2} s_{11}[n] \\
& +\left\{2 m_{2}^{2} \sum_{k}\left(\left|s_{11}[k]\right|^{2}+\left|s_{21}[k]\right|^{2}\right)\right. \\
& \left.\quad+c_{o}\left(2 m_{2}^{2}-m_{4}\right) \sum_{k}\left|s_{12}[k]\right|^{2}+m_{4}\right\} s_{11}[n]=0
\end{aligned}
$$

Therefore, at each stationary point of $C_{\mathrm{MIMO}}, s_{11}[n]$ must satisfy

$$
\left|s_{11}[n]\right|^{2}= \begin{cases}t_{11}, & \text { if } n \in I \\ 0, & \text { otherwise }\end{cases}
$$

where $t_{11}$ is a nonnegative number, and $I$ is a set consisting of finite number integers. Following Foschini's perturbation argument [6], at the minima of $C_{\mathrm{MIMO}}$

$$
\left|s_{11}[n]\right|^{2}=t_{11} \delta\left[n-n_{11}\right]
$$

for some integer $n_{11}$. Since $C_{\mathrm{MIMO}}$ is symmetric on $s_{i j}[n]$, the necessary condition for $C_{\text {MIMO }}$ to attain its minima is

$$
\left|s_{i j}[n]\right|^{2}=t_{i j} \delta\left[n-n_{i j}\right]
$$

for $i=1,2$, where $t_{i j}$ 's, for $i, j=1,2$, are some nonnegative real numbers, and $n_{i j}$ 's, for $i, j=1,2$, are some integers.

Substituting (55) into (47) and applying Lemma 3, we obtain the only possible minima of $C_{\text {MIMO }}$ are

$$
\begin{aligned}
\left|s_{11}\left[n+n_{11}\right]\right|^{2} & =\left|s_{22}\left[n+n_{22}\right]\right|^{2}=\delta[n] \\
\left|s_{12}[n]\right|^{2} & =\left|s_{21}[n]\right|^{2}=0
\end{aligned}
$$

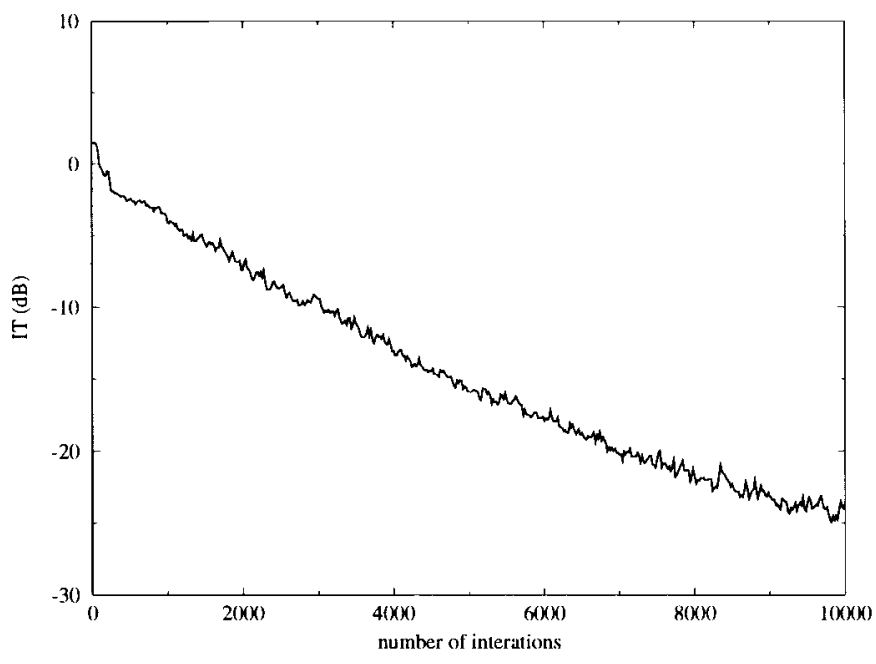

Fig. 4. Convergence of the MIMO-CMA blind equalizer.

or

$$
\begin{aligned}
\left|s_{11}[n]\right|^{2} & =\left|s_{22}[n]\right|^{2}=0 \\
\left|s_{12}\left[n+n_{12}\right]\right|^{2} & =\left|s_{21}\left[n+n_{21}\right]\right|^{2}=\delta[n]
\end{aligned}
$$

for some integers $n_{11}, n_{12}, n_{21}$, and $n_{22}$. When $s_{i j}[n]$ satisfies (56) or (57), both $C\left(y_{1}[n]\right)$ and $C\left(y_{2}[n]\right)$ reach their global minima, and $-c_{o} K\left(y_{1}, y_{2}\right)$ also reaches its global minimum, therefore, $C_{\mathrm{MIMO}}$ attains its global minimum. Therefore, all minima of $C_{\text {MIMO }}$ are global ones, which implies that the MIMO equalizer will converge to one of its global minima regardless of its initial setting.

ii) Assume the MIMO system and equalizer satisfy condition ii) in the theorem.

Define the equalizer parameter matrix as

$$
\mathcal{G}_{K} \triangleq(G[0], G[1], \cdots, G[K-1])
$$

and the MIMO equalized system parameter matrix as

$$
\mathcal{S}_{L+K-1} \triangleq(S[0], S[1], \cdots, S[L+K-2]) .
$$

Then

$$
\mathcal{S}_{L+K-1}=\mathcal{G}_{K} \mathcal{H}_{K}
$$

According to Lemma $1, \mathcal{H}_{K}$ for $K \geq d L-1$ is of full column rank. Hence, for any $\mathcal{S}_{L+K-1} \in \mathcal{C}^{2 \times 2(L+K-1)}$, there is an MIMO equalizer matrix $\mathcal{G}_{K}=\mathcal{S}_{L+K-1}\left(\mathcal{H}_{K}^{\dagger} \mathcal{H}\right)^{-1} \mathcal{H}_{K}^{\dagger}$ satisfying (60). Using similar arguments to the proof of the first part, we are able to obtain that the possible minima of the cost function $C_{\text {MIMO }}$ on $\mathcal{C}^{2 \times 2(L+K-1)}$ are

$$
\begin{aligned}
\left|s_{11}\left[n+n_{11}\right]\right|^{2} & =\left|s_{22}\left[n+n_{22}\right]\right|^{2}=\delta[n] \\
\left|s_{12}[n]\right|^{2} & =\left|s_{21}[n]\right|^{2}=0 \\
\left|s_{11}[n]\right|^{2} & =\left|s_{22}[n]\right|^{2}=0 \\
\left|s_{12}\left[n+n_{12}\right]\right|^{2} & =\left|s_{21}\left[n+n_{21}\right]\right|^{2}=\delta[n]
\end{aligned}
$$

for $0 \leq n_{i j} \leq L+K-2$ and they are all global ones. Hence, the MIMO equalizer in this case will converge to one of the global minima regardless of its initial condition.

From Theorem 4, the new blind algorithm is able to recover all input signals simultaneously. Furthermore, the MIMO FIR 

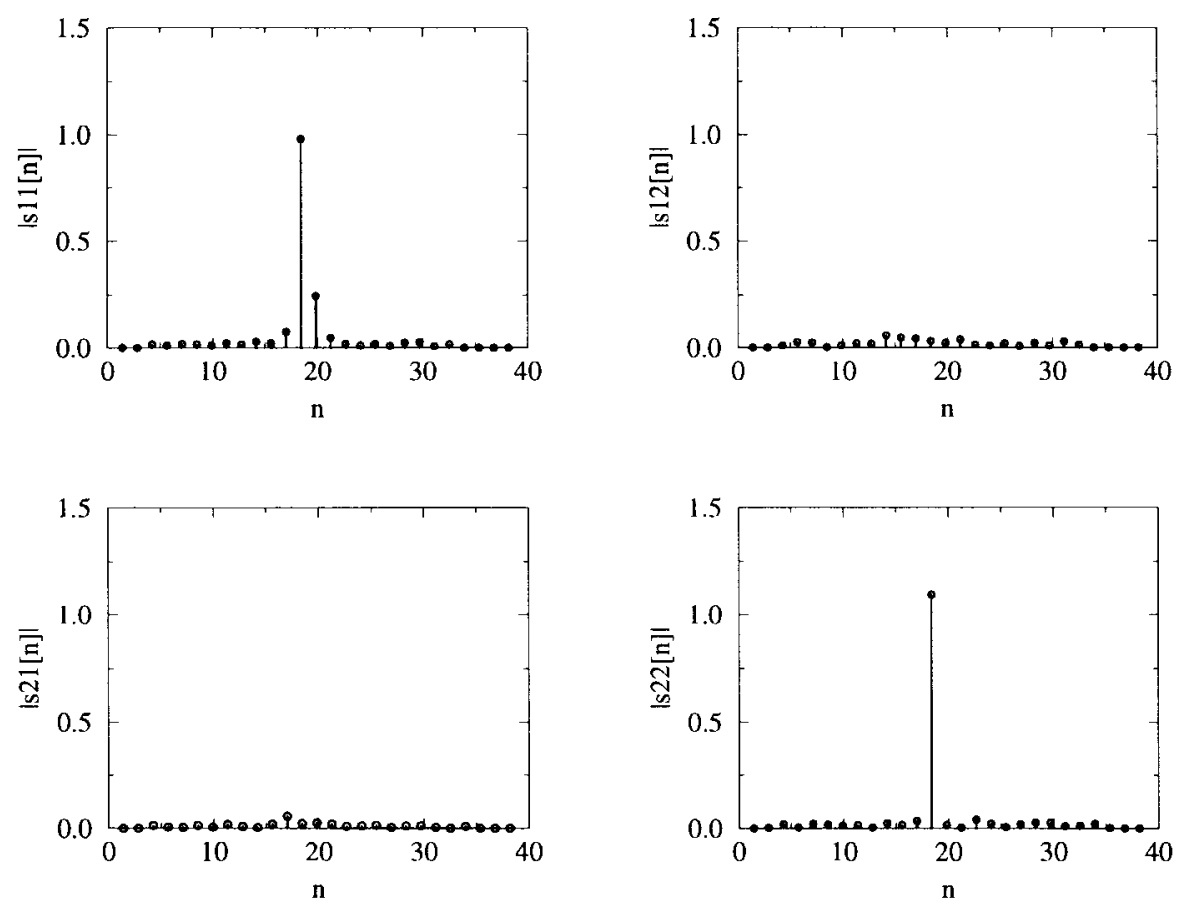

Fig. 5. The impulse responses of the equalized system after 20000 iterations.

system satisfying certain conditions can be perfectly equalized by MIMO FIR equalizers employing the new algorithm. Hence, the new blind algorithm is ideal for source separation and distortion compensation of MIMO systems.

\section{B. Local Convergence}

Similar to the CMA for SISO systems, the new MIMO blind algorithm also suffers from the local convergence problem if the MIMO system and equalizers does not satisfy either of the two conditions in Theorem 4.

To construct an example demonstrating such a problem, let us consider a 2-input/2-output AR system with the transfer functions

$$
\begin{aligned}
h_{11}(z)=h_{22}(z)=\frac{1}{1-z^{-1} \alpha}, 0<|\alpha| & <\frac{1}{\sqrt{3}}, \text { and } \\
h_{12}(z) & =h_{21}(z)=0 .
\end{aligned}
$$

Using Theorem 1, it is easy to check that $H(z)$ satisfies the distortionless reception condition. If an MIMO FIR equalizer with length $K=2$ is used, then

$$
G(z)=\left(\begin{array}{cc}
1-\alpha z^{-1} & 0 \\
0 & 1-\alpha z^{-1}
\end{array}\right)
$$

can perfectly equalize this MIMO system. However, as discussed in [13], there is a $\gamma \neq 0$ such that

$$
g_{11}[n]=g_{22}[n]=\gamma \delta[n-1] \quad g_{12}[n]=g_{21}[n]=0
$$

is a local minimum of both $C\left(y_{1}[n]\right)$ and $C\left(y_{2}[n]\right)$. Since $-c_{o} K\left(y_{1}, y_{2}\right)=0$ in this case that is also a minimum point of $-c_{o} K\left(y_{1}, y_{2}\right)$. Therefore, the $g_{i j}[n]$ in (64) is a local minimum point of $C_{\mathrm{MIMO}}$.

\section{COMPuter Simulations}

In order to confirm the analysis results and illustrate the effectiveness of the proposed algorithm, we present two computer simulation examples.

In our simulations, the system inputs $a_{i}[n]$ 's are independent for different $i$ 's or $n$ 's, and they are uniformly distributed over $\{ \pm 1 / \sqrt{2}, \pm \jmath / \sqrt{2}\}$. The system noise is complex white Gaussian with zero mean and variance determined by the signal-to-noise ratio (SNR) of systems.

\section{A. Convergence of the MIMO-CMA Blind Equalizer}

In this simulation example, the impulse response of a 2-input/3-output FIR system is given by

$$
\begin{gathered}
H[0]=\left(\begin{array}{cc}
-1.9522 & -0.5706 \\
-0.5666 & 0.4246 \\
-1.1293 & 0.7666
\end{array}\right) \text { and } \\
H[1]=\left(\begin{array}{cc}
1.0691 & -1.8841 \\
-0.7926 & 0.0598 \\
0.3569 & -0.2744
\end{array}\right)
\end{gathered}
$$

and $\mathrm{SNR}=30 \mathrm{~dB}$ at the system outputs.

An MIMO-CMA FIR equalizer is used for the MIMO FIR system. The length of the equalizer is $K=3$ with initial setting $g_{m}[n]=\delta[m-1, n-1]$ and the step-size $\mu=0.0005$. Fig. 3 is the impulse responses of the equalized system after 10000 iterations (symbols). From this figure, the MIMO-CMA FIR equalizer is able to recover the second system input, mitigate the ISI, and suppress the first system input. Fig. 4 illustrates the variation of interference during iterations. The interference, IT, here includes intersymbol interference and 

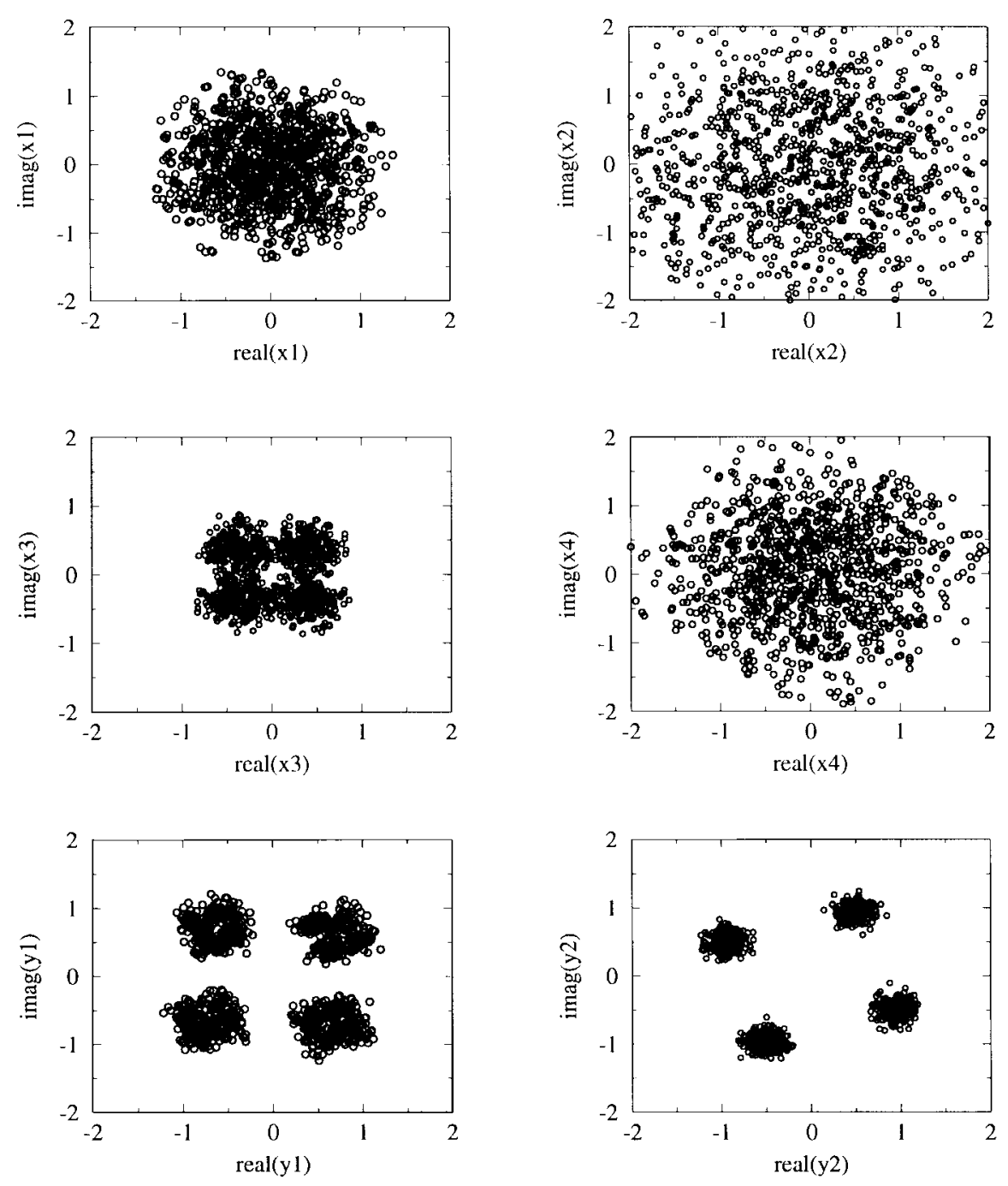

Fig. 6. 1000 inputs $x_{1}[n], x_{2}[n], x_{3}[n]$, and $x_{4}[n]$ and outputs $y_{1}[n], y_{2}[n]$ of the new blind equalizer after 20000 iterations.

interference from the other sources, and is defined as

$$
\mathrm{IT}=\frac{\sum_{i, n}\left|s_{i}[n]\right|^{2}-\max _{i, n}\left|s_{i}[n]\right|^{2}}{\max _{i, n}\left|s_{i}[n]\right|^{2}} .
$$

The simulation results in these two figures confirm Theorem 3.

\section{B. Convergence of the New MIMO Blind Equalizer}

In this simulation example, the impulse responses of a 2input/4-output FIR system is shown as in Table II, and SNR = $30 \mathrm{~dB}$.

The equalizer length used in our simulation is 20 with initial settings $g_{11}[n]=g_{22}[n]=\delta[n-10]$. The other equalizer parameters are $\mu=0.0001, \epsilon=0.01$, and $c_{o}=1.2$.

Fig. 5 shows the impulse response $s_{i j}[n]$ of the equalized system after 20000 iterations. Fig. 6 demonstrates the constellations of 1000 system outputs and 1000 equalizer outputs. Fig. 7 illustrates the interference of the equalizer outputs, defined as

$$
\mathrm{IT}_{j}=\frac{\sum_{i, n}\left|s_{i j}[n]\right|^{2}-\max _{i, n}\left|s_{i j}[n]\right|^{2}}{\max _{i, n}\left|s_{i j}[n]\right|^{2}} .
$$

According to Fig. 5, the two sources are separated and the distortions are compensated. The first equalizer output recovers the first source and the second output recovers the second source.

\section{CONCLUSION}

This paper investigates blind source separation and equalization of multiple-input/multiple-output systems. We have demonstrated that the MIMO-CMA equalizer is able to recover a signal from one source, compensate for system distortion, and suppress the signals from other sources. To recover signals from all sources simultaneously and compensate for system distortion, a novel blind equalization algorithm for MIMO systems is proposed. Its global convergence is illustrated theoretically and by computer simulations. The proposed al- 
TABLE II

IMPULSE RESPONSES OF A 2-INPUT/4-OUTPUT SySTEM

\begin{tabular}{|c|c||c|c|c|c|}
\hline $\mathrm{i}$ & $\mathrm{n}$ & $h_{1 i}[n]$ & $h_{2 i}[n]$ & $h_{3 i}[n]$ & $h_{4 i}[n]$ \\
\hline \hline & 0 & $-0.0000-\jmath 0.0000$ & $0.0000-\jmath 0.0000$ & $-0.0000-\jmath 0.0000$ & $-0.0000+\jmath 0.0000$ \\
& 1 & $-0.1700-\jmath 1.0300$ & $0.7100-\jmath 1.0900$ & $0.2100-\jmath 0.1000$ & $-0.5700+\jmath 0.8000$ \\
1 & 2 & $0.4700-\jmath 0.6200$ & $-0.5200-\jmath 0.6500$ & $1.0100-\jmath 0.0300$ & $-0.2900+\jmath 0.7000$ \\
& 3 & $0.0000+\jmath 0.0000$ & $-0.0000+\jmath 0.0000$ & $0.0000+\jmath 0.0000$ & $0.0000+\jmath 0.0000$ \\
& 4 & $-0.0000+\jmath 0.0000$ & $0.0000+\jmath 0.0000$ & $-0.0000-\jmath 0.0000$ & $0.0000-\jmath 0.0000$ \\
& 5 & $0.0000-\jmath 0.0000$ & $-0.0000-\jmath 0.0000$ & $0.0000-\jmath 0.0000$ & $-0.0000+\jmath 0.0000$ \\
\hline \hline & 0 & $0.0125-\jmath 0.0681$ & $-0.0755+\jmath 0.2306$ & $-0.0000-\jmath 0.0000$ & $-0.0000+\jmath 0.0000$ \\
& 1 & $-0.0590+\jmath 0.5046$ & $0.4355-\jmath 1.6674$ & $0.2100-\jmath 0.1000$ & $-0.5700+\jmath 0.8000$ \\
2 & 2 & $0.0586+\jmath 0.1737$ & $-0.1390-\jmath 0.4800$ & $1.0100-\jmath 0.0300$ & $-0.2900+\jmath 0.7000$ \\
& 3 & $0.2915+\jmath 0.1841$ & $-0.9865-\jmath 0.2353$ & $0.0000+\jmath 0.0000$ & $0.0000+\jmath 0.0000$ \\
& 4 & $-0.0418-\jmath 0.0185$ & $0.1448+\jmath 0.0087$ & $-0.0000-\jmath 0.0000$ & $0.0000-\jmath 0.0000$ \\
& 5 & $0.0144+\jmath 0.0112$ & $-0.0479-\jmath 0.0181$ & $0.0000-\jmath 0.0000$ & $-0.0000+\jmath 0.0000$ \\
\hline
\end{tabular}

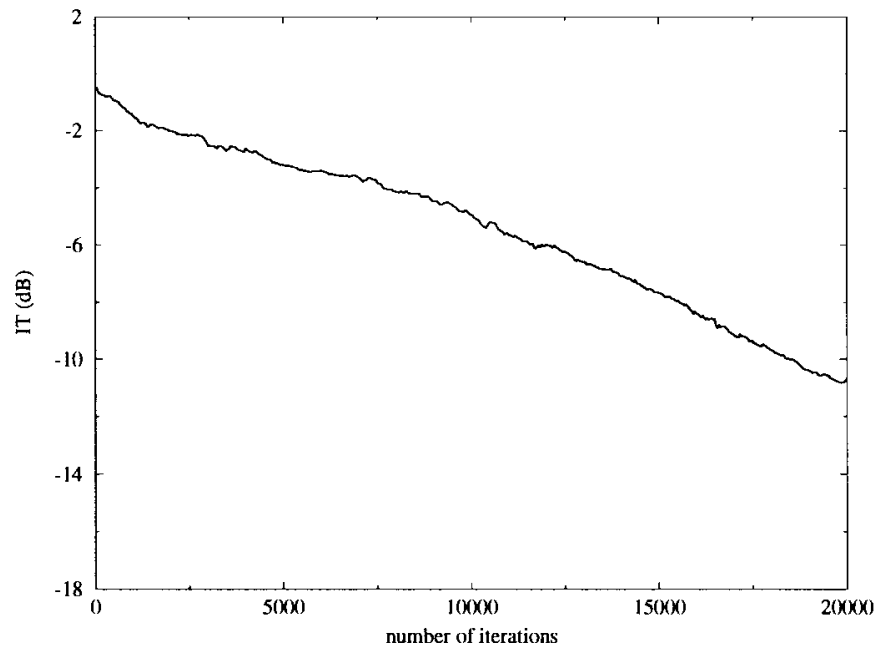

(a)

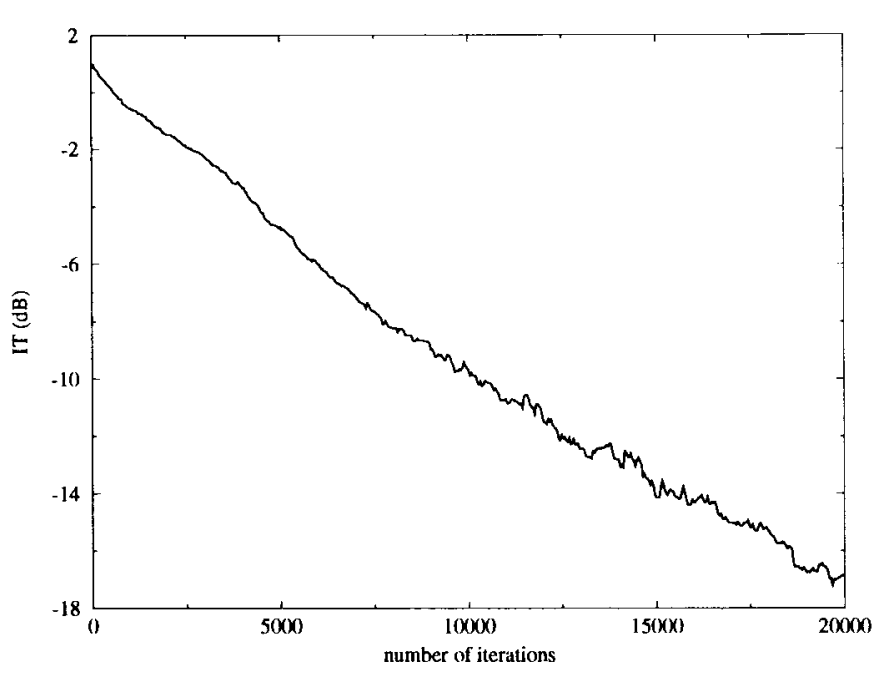

(b)

Fig. 7. Convergence of the new algorithm for the MIMO channel. (a) IT for $y_{1}[n]$. (b) IT for $y_{2}[n]$.

gorithm can be applied not only in multiple signal separation in array processing, but also in diverse fields of engineering including speech processing, data communication, sonar array processing, and in the analysis of biological systems.

\section{APPENDIX A}

\section{PROOF OF LEMMA 2}

According to the definition in (36), we have

$$
\begin{aligned}
\operatorname{Cum} & \left(y_{1}[n], y_{1}^{*}[n], y_{2}[n+k], y_{2}^{*}[n+k]\right) \\
= & \operatorname{Cum}\left(\sum_{i=1}^{2} \sum_{l} a_{i}[l] s_{i 1}[n-l], \sum_{i=1}^{2} \sum_{l} a_{i}^{*}[l] s_{i 1}^{*}[n-l],\right. \\
& \left.\sum_{i=1}^{2} \sum_{l} a_{i}[l] s_{i 2}[n+k-l], \sum_{i=1}^{2} \sum_{l} a_{i}^{*}[l] s_{i 2}^{*}[n+k-l]\right) \\
= & \sum_{i_{1}, i_{2}, i_{3}, i_{4}=1} \sum_{l_{1}, l_{2}, l_{3}, l_{4}} s_{i_{1} 1}\left[n-l_{1}\right] s_{i_{2} 1}^{*}\left[n-l_{2}\right] \\
& \times s_{i_{3} 2}\left[n+k-l_{3}\right] s_{i_{4} 2}^{*}\left[n+k-l_{4}\right] \\
& \times \operatorname{Cum}\left(a_{i_{1}}\left[l_{1}\right], a_{i_{2}}^{*}\left[l_{2}\right], a_{i_{3}}\left[l_{3}\right], a_{i_{4}}^{*}\left[l_{4}\right]\right) .
\end{aligned}
$$

Since we have assumed $a_{i}[n]$ 's are independent for different $i$ or $n$, then

$$
\begin{gathered}
\operatorname{Cum}\left(a_{i_{1}}\left[l_{1}\right], a_{i_{2}}^{*}\left[l_{2}\right], a_{i_{3}}\left[l_{3}\right], a_{i_{4}}^{*}\left[l_{4}\right]\right) \\
\quad= \begin{cases}m_{4}-2 m_{2}^{2}, & \text { for } i_{1}=i_{2}=i_{3}=i_{4} \\
0, & \text { and } l_{1}=l_{2}=l_{3}=l_{4}\end{cases}
\end{gathered}
$$

Therefore,

$$
\begin{aligned}
& \operatorname{Cum}\left(y_{1}[n], y_{1}^{*}[n], y_{2}[n+k], y_{2}^{*}[n+k]\right) \\
& =\left(m_{4}-2 m_{2}^{2}\right) \sum_{i=1,2} \sum_{n=-\infty}^{\infty}\left|s_{i 1}[n]\right|^{2}\left|s_{i 2}[n+k]\right|^{2} .
\end{aligned}
$$

From (A.3), (36) can be expressed as

$$
K\left(y_{1}, y_{2}\right)=\frac{1}{2}\left(m_{4}-2 m_{2}^{2}\right) \sum_{i=1,2} \prod_{j=1,2}\left(\sum_{k}\left|s_{i j}[k]\right|^{2}\right) .
$$




$$
\nabla^{2} f=\frac{1}{2}\left(\begin{array}{cccc}
m_{4} & 2 m_{2}^{2} & c_{o}\left(2 m_{2}^{2}-m_{4}\right) & 0 \\
2 m_{2}^{2} & m_{4} & 0 & c_{o}\left(2 m_{2}^{2}-m_{4}\right) \\
c_{o}\left(2 m_{2}^{2}-m_{4}\right) & 0 & m_{4} & 2 m_{2}^{2} \\
0 & c_{o}\left(2 m_{2}^{2}-m_{4}\right) & 2 m_{2}^{2} & m_{4}
\end{array}\right) \text {. }
$$

$$
\begin{aligned}
& f\left(\epsilon_{11}, 1+\epsilon_{12}, 1+\epsilon_{21}, \epsilon_{22}\right)-f(0,1,1,0) \\
& =\frac{1}{4}\left\{2\left(1+c_{o}\right)\left(2 m_{2}^{2}-m_{4}\right) \epsilon_{11}+m_{4} \epsilon_{11}^{2}+2 c_{o}\left(2 m_{2}^{2}-m_{4}\right) \epsilon_{11} \epsilon_{12}+4 m_{2}^{2} \epsilon_{11} \epsilon_{21}+m_{4} \epsilon_{21}^{2}\right. \\
& \left.+2\left(1+c_{o}\right)\left(2 m_{2}^{2}-m_{4}\right) \epsilon_{22}+m_{4} \epsilon_{22}^{2}+2 c_{o}\left(2 m_{2}^{2}-m_{4}\right) \epsilon_{22} \epsilon_{21}+4 m_{2}^{2} \epsilon_{22} \epsilon_{12}+m_{4} \epsilon_{12}^{2}\right\} \\
& \geq \frac{1}{4}\left\{\frac{2\left(1+c_{o}\right)\left(2 m_{2}^{2}-m_{4}\right)\left[2 m_{2}^{2}+m_{4}+c_{o}\left(2 m_{2}^{2}-m_{4}\right)\right]}{2 m_{4}} \epsilon_{11}^{2}\right. \\
& +m_{4} \epsilon_{11}^{2}+2 c_{o}\left(2 m_{2}^{2}-m_{4}\right) \epsilon_{11} \epsilon_{12}+4 m_{2}^{2} \epsilon_{11} \epsilon_{21}+m_{4} \epsilon_{21}^{2} \\
& +\frac{2\left(1+c_{o}\right)\left(2 m_{2}^{2}-m_{4}\right)\left[2 m_{2}^{2}+m_{4}+c_{o}\left(2 m_{2}^{2}-m_{4}\right)\right]}{2 m_{4}} \epsilon_{22}^{2} \\
& \left.+m_{4} \epsilon_{22}^{2}+2 c_{\circ}\left(2 m_{2}^{2}-m_{4}\right) \epsilon_{22} \epsilon_{21}+4 m_{2}^{2} \epsilon_{22} \epsilon_{12}+m_{4} \epsilon_{12}^{2}\right\} \\
& =\frac{1}{4}\left\{\frac{2 m_{2}^{2} m_{4}}{2 m_{2}^{2}+c_{o}\left(2 m_{2}^{2}-m_{4}\right)}\left(\frac{2 m_{2}^{2}+c_{o}\left(2 m_{2}^{2}-m_{4}\right)}{m_{4}} \epsilon_{11}+\epsilon_{21}\right)^{2}\right. \\
& +\frac{c_{o}\left(2 m_{2}^{2}-m_{4}\right) m_{4}}{2 m_{2}^{2}+c_{o}\left(2 m_{2}^{2}-m_{4}\right)}\left(\frac{2 m_{2}^{2}+c_{o}\left(2 m_{2}^{2}-m_{4}\right)}{m_{4}} \epsilon_{11}+\epsilon_{12}\right)^{2} \\
& +\frac{2 m_{2}^{2} m_{4}}{2 m_{2}^{2}+c_{o}\left(2 m_{2}^{2}-m_{4}\right)}\left(\frac{2 m_{2}^{2}+c_{o}\left(2 m_{2}^{2}-m_{4}\right)}{m_{4}} \epsilon_{22}+\epsilon_{12}\right)^{2} \\
& \left.+\frac{c_{o}\left(2 m_{2}^{2}-m_{4}\right) m_{4}}{2 m_{2}^{2}+c_{o}\left(2 m_{2}^{2}-m_{4}\right)}\left(\frac{2 m_{2}^{2}+c_{o}\left(2 m_{2}^{2}-m_{4}\right)}{m_{4}} \epsilon_{22}+\epsilon_{21}\right)^{2}\right\} \\
& \geq 0
\end{aligned}
$$

\section{APPENDIX B}

PROOF OF LEMMA 3

Direct calculation yields (B.1) at the top of this page. Since $\nabla^{2} f\left(t_{11}, t_{12}, t_{21}, t_{22}\right)$ is not nonnegative-definite, from [20, Proposition 2, pp. 174], $f\left(t_{11}, t_{12}, t_{21}, t_{22}\right)$ has no minimum inside $[0,+\infty)^{4}$. Therefore, its possible minima must be on the boundary of $[0,+\infty)^{4}$.

Without loss of generality, assume $t_{22}=0$ and let

$$
f_{1}\left(t_{11}, t_{12}, t_{21}\right)=f\left(t_{11}, t_{12}, t_{21}, 0\right) \text {. }
$$

The minimum of $f_{1}\left(t_{11}, t_{12}, t_{21}\right)$ is $\left(t_{11}, t_{12}, t_{21}\right)=(0,1,1)$ when $c_{o}>m_{4} /\left(2 m_{2}^{2}-m_{4}\right)$. Therefore,

$$
\left(t_{11}, t_{12}, t_{21}, t_{22}\right)=(0,1,1,0)
$$

may be a minimum of $f\left(t_{11}, t_{12}, t_{21}, t_{22}\right)$ on $[0,+\infty)^{4}$. For any $\epsilon_{11}, \epsilon_{12}, \epsilon_{21}, \epsilon_{22}$ satisfying

$$
0 \leq \epsilon_{11}, \epsilon_{22}<\frac{2 m_{4}}{2 m_{2}^{2}+m_{4}+c_{o}\left(2 m_{2}^{2}-m_{4}\right)}
$$

we have (B.2) at the top of this page. Hence,

$$
\left(t_{11}, t_{12}, t_{21}, t_{22}\right)=(0,1,1,0)
$$

is a minimum of $f\left(t_{11}, t_{12}, t_{21}, t_{22}\right)$ on $[0,+\infty)^{4}$.

Because of the symmetry of $f\left(t_{11}, t_{12}, t_{21}, t_{22}\right)$

$$
\left(t_{11}, t_{12}, t_{21}, t_{22}\right)=(1,0,0,1)
$$

is another minimum of $f\left(t_{11}, t_{12}, t_{21}, t_{22}\right)$.

\section{REFERENCES}

[1] K. Abed-Meriam, P. Loubaton, and E. Moulines, "A subspace algorithm for certain blind identification problems," IEEE Trans. Inform. Theory, vol. 43, pp. 499-511, Mar. 1997.

[2] K. Abed-Meriam, W. Qiu, and Y. Hua, "Blind system identification," Proc. IEEE, pp. 1310-1322, vol. 85, Aug. 1997.

[3] A. Benveniste, M. Goursat, and G. Ruget, "Robust identification of a nonminimum phase system: Blind adjustment of a linear equalizer in data communications," IEEE Trans. Automat. Contr., vol. AC-25, pp. 385-399, June 1980.

[4] Z. Ding, R. A. Kennedy, B. D. O. Anderson, and C. R. Johnson Jr. "Ill-convergence of Godard blind equalizers in data communication systems," IEEE Trans. Commun., vol. 39, pp. 459-472, Sept. 1991. 
[5] D. Donoho, "On minimum entropy deconvolution," in Applied Time Series Analysis II. New York: Academic, 1981, pp. 565-608.

[6] G. J. Foschini, "Equalization without altering or detect data," AT\&T Tech. J., pp. 1885-1911, Oct. 1985.

[7] R. D. Gitlin and S. B. Weinstein, "Fractionally-spaced equalization: An improved digital transversal equalizer," Bell Syst. Tech. J., vol. 60, pp. 275-296, Feb. 1981

[8] D. N. Godard, "Self-recovering equalization and carrier tracking in twodimensional data communication systems," IEEE Trans. Commun., vol. COM-28, pp. 1867-1875, Nov. 1980.

[9] A. Gorokhov and P. Loubaton, "MIMO ARMA systems: Second order blind identification for signal extraction," in Proc. 8th IEEE SSAP Workshop, Nov. 1996, pp. 348-351.

[10] T. Kailath, Linear Systems. Englewood Cliffs, NJ: Prentice-Hall, 1980

[11] J. P. LeBlanc, I. Fijalkow, B. Huber, and C. R. Johnson Jr., "Fractionally spaced CMA equalizers under periodic and correlated inputs," in Proc. ICASSP'95, May 1995, vol. 2, pp. 1041-1045.

[12] Y. (Geoffrey) Li, J. H. Winters, and N. R. Sollenberger, "Spatialtemporal equalization for IS-136 TDMA systems with rapid dispersive fading and co-channel interference," IEEE Trans. Veh. Technol., accepted for publication.

[13] Y. Li and Z. Ding, "Convergence analysis of finite length blind adaptive equalizers," IEEE Trans. Signal Processing, vol. 43, pp. 2120-2129, Sept. 1995.

[14] _ "Global convergence of fractionally spaced Godard equalizer," IEEE Trans. Signal Processing, vol. 44, pp. 818-826, Apr. 1996.

[15] Y. Li and K. J. R. Liu, "On blind MIMO channel identification using second order statistics," in Proc. 30th Conf. Information Sciences and Systems (Princeton, NJ, Mar. 1996).

[16] , "On blind equalization of MIMO channels," in Proc. of ICC'96 (Dallas, TX, June 1996), vol. 2, pp. 1020-1024.

[17] _, "Blind identification and equalization for multipleinput/multiple-output channels," in Proc. 1996 IEEE Global Telecommunications Conf. (London, U.K., Nov. 1996), pp. 17891793.

[18] Y. (Geoffrey) Li and K. J. R. Liu, "Blind identification and equalization for wireless communications using antenna array," in Proc. SPIE'97, Aug. 1997, vol. 3162, pp. 251-262.

[19] F. Ling and S. U. H. Qureshi, "Convergence and steady-state behavior of phase-splitting fractionally-spaced equalizer," IEEE Trans. Commun., vol. 38, pp. 418-425, Apr. 1990.

[20] D. G. Luenberger, Linear and Nonlinear Program, 2nd ed. Reading, MA: Addison-Wesley, 1984.

[21] S. Mayrargue, "A blind spatio-temporal equalizer for a radio-mobile channel using the constant modulus algorithm (CMA)," in Proc. IEEE ICASSP'94 (Adelaide, Australia, Apr. 1994), pp. III 317-320.

[22] C. L. Nikias, "ARMA bispectrum approach to nonminimum phase system identification," IEEE Trans. Acoust., Speech, Signal Processing, vol. 36, pp. 513-525, Apr. 1988.

[23] C. B. Papadia and A. J. Paulraj, "A constant modulus algorithm for multiuser signal separation in presence of delay spread using antenna arrays," IEEE Signal Processing Lett., vol. 4, pp. 178-181, June 1997.

[24] B. R. Petersen and D. D. Falconer, "Suppression of adjacent-channel, co-channel, and intersymbol interference by equalizers and linear combiners," IEEE Trans. Commun., vol. 42, pp. 3109-3118, Dec. 1994.
[25] Y. Sato, "A method of self-recovering equalization for multi-level amplitude modulation," IEEE Trans. Commun., vol. COM-23, pp. 679-682, June 1975.

[26] O. Shalvi and E. Weinstein, "New criteria for blind deconvolution of non-minimum phase systems (channels)," IEEE Trans. Inform. Theory, vol. 36, pp. 312-321, Mar. 1990.

[27] D. Slock, "Blind joint equalization of multiple synchronous mobile users using oversampling and/or multiple antennas," in Proc. 28th Asilomar Conf. Signal Systems and Computers (Pacific Grove, CA, Oct. 1994), pp. $1154-1158$

[28] J. R. Treichler, V. Wolff, and C. R. Johnson Jr., "Observed misconvergence in the constant modulus adaptive algorithm," in Proc. 25th Asilomar Conf. Signals, Systems and Computers (Pacific Grove, CA, Oct. 1991), pp. 663-667.

[29] J. R. Treichler and B. G. Agee, "A new approach to multipath correction of constant modulus signals," IEEE Trans. Acoust., Speech Signal Processing, vol. ASSP-31, pp. 349-372, Apr. 1983.

[30] J. R. Treichler and M. G. Larimore, "New processing techniques based on the constant modulus adaptive algorithm," IEEE Trans. Acoust., Speech Signal Processing, vol. ASSP-33, pp. 420-431, Apr. 1985.

[31] _ "The tone capture properties of CMA-based interference suppressors," IEEE Trans. Acoust., Speech Signal Processing, vol. ASSP-33, pp. 946-958, Aug. 1985

[32] S. Talwar, M. Viberg, and A. Paulraj, "Blind separation of synchronous co-channel digital signals using an antenna array-Part I: Algorithms," IEEE Trans. Signal Processing, vol. 44, pp. 1184-1197, May 1996.

[33] J. K. Tugnait, "Identification of linear stochastic systems via second and fourth-order cumulant matching," IEEE Trans. Inform. Theory, vol. IT-33, pp. 393-407, May 1987.

[34] _ _ "On fractionally-spaced blind adaptive equalization under symbol timing offsets using Godard and related equalizers," in Proc ICASSP'95, May 1995, vol. 3, pp. 1976-1979.

[35] _ _ "Blind spatio-temporal equalization and impulse response estimation for MIMO channels using a Godard cost function," IEEE Trans. Signal Processing, vol. 45, pp. 268-271, Jan. 1997.

[36] G. Ungerboeck, "Fractional tap-spacing equalizer and consequences for clock recovery in data modems," IEEE Trans. Commun., vol. COM-24, pp. 856-864, Aug. 1976.

[37] A. van der Veen, S. Talwar, and A. Paulraj, "Blind estimation of multiple digital signals transmitted over FIR channels," IEEE Signal Processing Lett., vol. 2, May 1995

[38] A. van der Veen and A. Paulraj, "Blind estimation of multiple digital signals transmitted over FIR channels," IEEE Trans. Signal Processing, vol. 44, pp 1136-1155, May 1996.

[39] S. Verdú, B. D. O. Anderson, and R. A. Kennedy, "Blind equalization without gain identification," IEEE Trans. Inform. Theory, vol. 39, pp. 292-297, Jan. 1993

[40] E. Weinstein, A. V. Oppenheim, M. Feder, and J. R. Buck, "Iterative and sequential algorithms for multisensor signal enhancement," IEEE Trans. Signal Processing, vol. 42, pp. 846-859, Apr. 1994.

[41] E. Weinstein, A. Swami, G. Giannakis, and S. Shamsunder, "Multichannel ARMA processes," IEEE Trans. Signal Processing, vol. 42, pp. 898-913, Apr. 1994

[42] J. Yang and S. Roy, "On joint transmitter and receiver optimization for multiple-input-multiple output (MIMO) transmission systems," IEEE Trans. Commun., vol. 42, pp. 3221-3231, Dec. 1994.

[43] D. Yellin and E. Weinstein, "Criteria for multichannel signal separation," IEEE Trans. Signal Processing, vol. 42, pp. 2158-2168, Aug. 1994. 